\title{
Bottom spectroscopy on dynamical $2+1$ flavor domain wall fermion lattices with a relativistic heavy quark action
}

\author{
Min Li* \\ Department of Physics, Columbia University, New York, NY 10027, USA \\ E-mail: minxolee@phys.columbia.edu
}

\section{RBC and UKQCD collaborations}

\begin{abstract}
Following the successful application of the relativistic heavy quark(RHQ) action to the charmonium and charm-strange meson spectrum, we study here the bottom system to explore the validity of this method in a regime with larger heavy quark momenta. The spectrum is calculated using the same dynamical $2+1$ flavor, $24^{3} \times 64$ domain wall fermion lattice configurations generated by the RBC and UKQCD collaborations and used in the earlier charm study. The 3 parameters in the RHQ action are determined by matching to the experimental bottom-strange meson masses and extrapolated to the chiral limit from three different sea quark masses. We predict the bottomonium mass spectrum and compare it with experiment. A theoretical estimation is also carried out to understand the $O\left(a^{2} p^{2}\right)$ systematic errors found in the numerical study.
\end{abstract}

The XXVI International Symposium on Lattice Field Theory

July 14-192008

Williamsburg, Virginia, USA

${ }^{*}$ Speaker. 


\section{Introduction}

Heavy quark physics is a long standing challenge for lattice QCD due to the fact that the masses are large in current lattice units. Here $m a \ll 1$ is no longer true and terms containing $(m a)^{n}$ (with $a$ the lattice spacing) become significant. This problem must be addressed by using effective field theories since a direct simulation is beyond the current computation power. Various heavy quark effective actions were developed for different physical systems. See Refs. [1, 2, 3, 4] for reviews on this topic.

In Ref. [5] we studied the relativistic heavy quark(RHQ) action [6, 7, 8, 9] on charmonium and charm-strange systems, and found excellent accuracy for this application on charm quarks. We determined the RHQ parameters at the $1 \%$ level, predicted $\chi_{c 0}$ and $\chi_{c 1}$ masses with sub-percent accuracy, and determined the lattice scale on the $\beta=2.13,24^{3} \times 64$ lattice ensemble with an accuracy at least as good as that of other methods. In this proceeding we will continue to explore the three-parameter RHQ method in bottom systems, a regime with much larger heavy quark momenta. We start with the bottom-strange system to determine the RHQ parameters as it has smaller $a^{2} \vec{p}^{2}$ discretization errors since $p \sim \Lambda_{Q C D}$, and then calculate the bottomonium states. To our surprise the calculations on bottomonium states have errors around $30 \mathrm{MeV}$ compared to the experiment. An attempt to theoretically estimate the $O\left(a^{2} \vec{p}^{2}\right)$ errors expected in this numerical study is also carried out at the end.

We start by briefly introducing the framework we have been using before. The lattice form of the action, following the formulation proposed in $[8,9]$, can be written as:

$$
S=\sum_{n, n^{\prime}} \bar{\Psi}_{n}\left\{m_{0}+\gamma_{0} D_{0}-\frac{1}{2} a D_{0}^{2}+\zeta\left[\vec{\gamma} \cdot \vec{D}-\frac{1}{2} a(\vec{D})^{2}\right]-a \sum_{\mu \nu} \frac{i}{4} c_{P} \sigma_{\mu \nu} F_{\mu \nu}\right\}_{n n^{\prime}} \Psi_{n^{\prime}}
$$

In heavy quark system, the temporal covariant derivative $D_{0}$ is on the order of $m a$ but the spatial derivatives $D_{i}$ are of order $\Lambda_{Q C D} a$ or $\alpha_{s} m a$ depending on the system under investigation. We found that only three free parameters are needed in the action: $m_{0}, c_{P}$ and $\zeta$. If the parameters are correctly tuned, the action will have small cutoff effects: $O\left(\Lambda_{Q C D} a\right)^{2}$ for heavy-light systems and $O\left(\alpha_{s} m a\right)^{2}$ for heavy quarkonium.

The lattices used in this work are the dynamical $2+1$ flavor $24^{3} \times 64$ DWF lattice configurations generated by the RBC-UKQCD collaborations. For better statistics, we place sources at different time slices separately for each configuration. Binning the data does not give any significant change in the size of the errors, indicating the auto-correlation of the lattice configurations is negligible.

\begin{tabular}{cccccc}
\hline \hline volume & $L_{s}$ & $\left(m_{\text {sea }}, m_{s}\right)$ & Traj(step) & \# of configs & sources \\
\hline $24^{3} \times 64$ & 16 & $(0.005,0.04)$ & $900-6880(20)$ & 300 & 0,32 \\
$24^{3} \times 64$ & 16 & $(0.01,0.04)$ & $1460-5060(40)$ & 91 & $0,16,32,48$ \\
$24^{3} \times 64$ & 16 & $(0.02,0.04)$ & $1885-3605(20)$ & 87 & $0,16,32,48$ \\
\hline
\end{tabular}

\section{Determination of the RHQ action}

To determine the action in a way in which errors are controlled, the parameters are fixed by matching physical observables, sensitive to those parameters, to their experimental or theoretical values. These parameters are determined for each ensemble with three different light sea 
quark masses and extrapolated to the chiral limit. In addition to mass combinations of pseudoscalar $\left(\eta_{b}, B_{s}\right)$, vector $\left(\Upsilon, B_{s}^{*}\right)$, scalar $\left(\chi_{b 0}\right)$ and axial-vector $\left(\chi_{b 1}\right)$ mesons in heavy-heavy (hh) and heavy-strange (hs) systems [10], we also calculate the tensor state $\left(h_{b}\right)$ for heavy-heavy system. Specifically we compute:

- Spin-averaged: $m_{s a}^{h h}=\frac{1}{4}\left(m_{\eta_{b}}+3 m_{\Upsilon}\right), m_{s a}^{h l}=\frac{1}{4}\left(m_{B_{s}}+3 m_{B_{s}^{*}}\right)$

- Hyperfine splitting: $m_{h s}^{h h}=m_{\Upsilon}-m_{\eta_{b}}, m_{h s}^{h l}=m_{B_{s}^{*}}-m_{B_{s}}$

- Mass ratio: $\frac{m_{1}}{m_{2}}$, where $E^{2}=m_{1}^{2}+\frac{m_{1}}{m_{2}} p^{2}, m_{1}$ : rest mass, $m_{2}$ : kinetic mass.

- Spin-orbit averaged and splitting (hh): $m_{s o s}^{h h}=m_{\chi_{b 1}}-m_{\chi_{b 0}}, m_{s o a}^{h h}=\frac{1}{4}\left(m_{\chi_{b 0}}+3 m_{\chi_{b 1}}\right)$

- Heavy-heavy ${ }^{1} P_{1}$ state $h_{b}$

Here are some sample plots of the bottomonium $\left(\eta_{b}\right)$ and bottom-strange $\left(B_{s}\right)$ pseudo-scalar correlators. One should notice the correlators are falling about 70 orders of magnitude for bottomonium and about 40 for bottom strange. We must be careful that the heavy propagators are sufficiently accurate when the time is large.
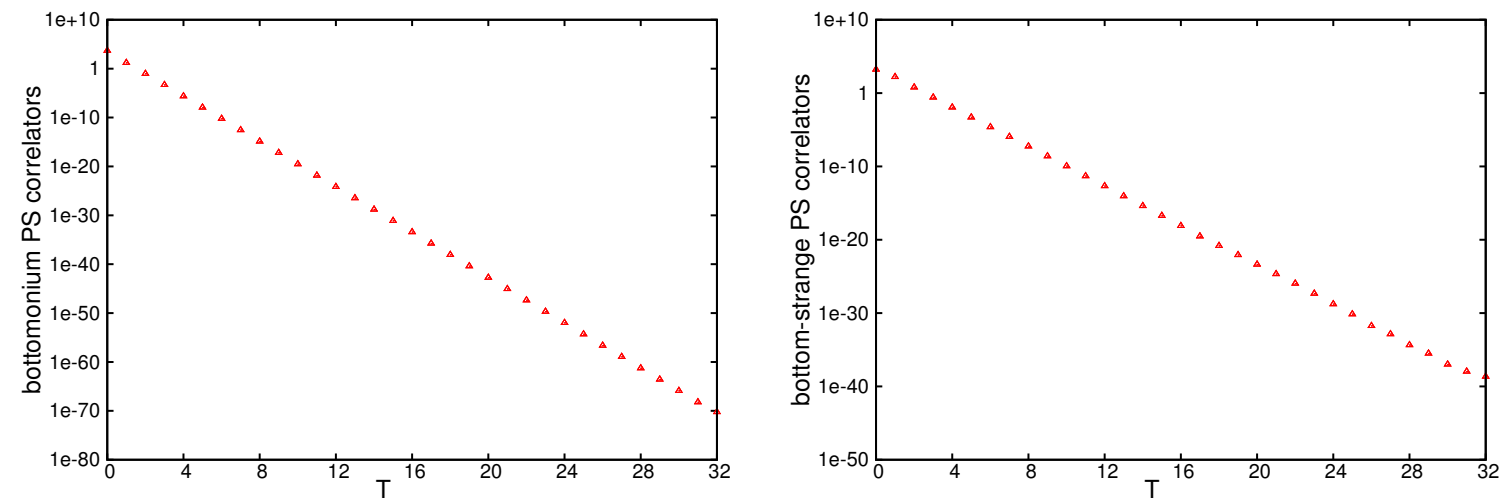

Figure 1: sample correlators of bottomonium and bottom-strange pseudo-scalar.

The matching method is the same as used in Ref. [5]. We again use the linear ansatz relating the three parameters $\left(X_{R H Q}\right)$ and the corresponding measured quantities $(Y(a))$ and determine the parameters by minimizing the $\chi^{2}$ defined in that reference. The linear approximation only holds in a certain region of the parameter space, which we estimate from our earlier work using a $16^{3} \times 32$ lattice. The linear coefficients are calculated directly using finite differences from seven point Cartesian parameter sets, which are centered at $\{7.3,4.0,4.3\}$ and with extent $\{0.5,1.0,0.3\}$. We examine the linearity of the parameter dependence in this region and required that the finally matched RHQ parameters actually lie within the region examined.

\subsection{Numerical details}

For all the calculations we use a box source with size 4, which is not optimized to give the best plateau. The physical strange quark mass used in the bottom-strange spectrum is 0.034 in lattice units [11]. Some sample effective mass plots for different states are shown for the $m_{\text {sea }}=0.005$ ensemble, Fig 2 and 3(left). We can see from the plots that the plateau for $\chi_{b 0}, \chi_{b 1}$ and $h_{b}$ states 
are not quite clear so their masses are subject to more systematic errors. All meson correlators are fit to a single state with exponential decay. The fitting ranges are chosen from an examination of the plateau in the effective mass plots, and they are summarized below in Tab. 1.

\begin{tabular}{|c|c|c|c|}
\hline fitting range & $\eta_{b} / \Upsilon$ & $\chi_{b 0} / \chi_{b 1} / h_{b}$ & $B_{s} / B_{s}^{*}$ \\
\hline$m_{\text {sea }}=0.005$ & {$[14,30]$} & {$[5,12]$} & {$[10,25]$} \\
$m_{\text {sea }}=0.01$ & {$[11,30]$} & {$[5,9]$} & {$[10,25]$} \\
$m_{\text {sea }}=0.02$ & {$[11,30]$} & {$[6,15]$} & {$[13,18]$} \\
\hline
\end{tabular}

Table 1: The time fitting ranges for different mesons at different sea quark masses.

The momentum dependence is studied for both the $\eta_{b}$ and $\Upsilon$ mesons and the mass ratio $m_{1} / m_{2}$ extracted from each are quite consistent. We use results from the $\Upsilon$ momentum dependence with the three lowest momenta. Please note that although we are using bottom-strange spectrum to fix the RHQ parameters, the mass ratio $m_{1} / m_{2}$ is determined from bottomonium because it is much better determined than it is from bottom-strange. To ensure the precision of the heavy quark propagator, we use an extremely tight CG stopping condition of $10^{-60}$. The CG stopping conditions are set to $10^{-8}$ for light quark propagators as before.
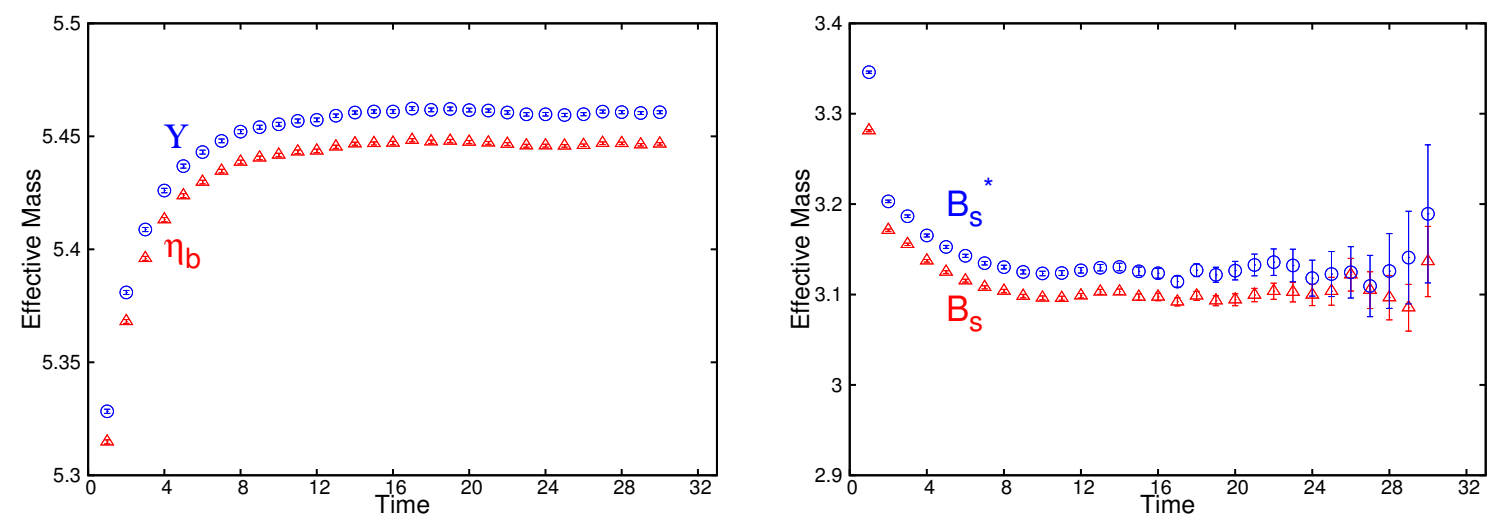

Figure 2: effective masses for the mesons on $m_{\text {sea }}=0.005$ ensembles: (left) $\eta_{b}$ and $\Upsilon$; (right) $B_{s}$ and $B_{s}^{*}$.
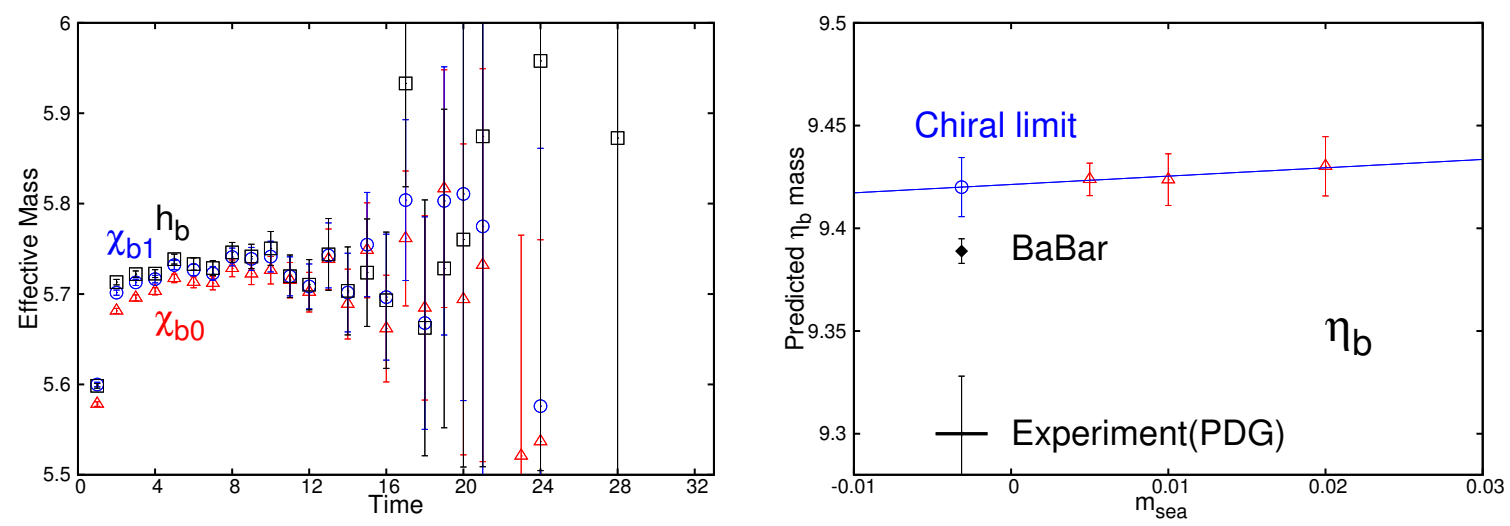

Figure 3: (left) Effective masses for the mesons: $\chi_{b 0}, \chi_{b 1}$ and $h_{b}$. (right) the prediction of $\eta_{b}$ mass in the chiral limit compared with the experiment. 


\section{Analysis and Results}

Again we list all the quantities calculated in this work: (1) $\frac{m_{1}}{m_{2}}(2) m_{B_{s}^{*}}-m_{B_{s}}(3) \frac{1}{4}\left(m_{B_{s}}+3 m_{B_{s}^{*}}\right)$ (4) $m_{\Upsilon}-m_{\eta_{b}}(5) \frac{1}{4}\left(m_{\eta_{b}}+3 m_{\Upsilon}\right)(6) m_{\chi_{b 1}}-m_{\chi_{b 0}}(7) \frac{1}{4}\left(m_{\chi_{b 0}}+3 m_{\chi_{b 1}}\right)(8) m_{h_{b}}$

\subsection{Heavy-strange sector: the RHQ action parameters}

As we mentioned before, except for the mass ratio $m_{1} / m_{2}$ which is determined from the $\Upsilon$ momentum dependence, only the heavy-light, $B_{s}$ and $B_{s}^{*}$ masses are used to fix the RHQ action parameters for the bottom system. Throughout the analysis, the lattice scale is taken to be $a^{-1}=$ $1.73 \mathrm{GeV}$ [12]. From matching to quantities (1)-(3), the RHQ parameters and the corresponding chiral limit extrapolations are shown in Tab. 2.

\begin{tabular}{|cccc|}
\hline \hline$m_{\text {sea }}$ & $m_{0} a$ & $c_{P}$ & $\zeta$ \\
\hline 0.005 & $7.37(7)$ & $3.84(40)$ & $4.21(3)$ \\
\hline 0.01 & $7.28(9)$ & $3.28(40)$ & $4.21(3)$ \\
\hline 0.02 & $7.30(11)$ & $3.52(53)$ & $4.24(4)$ \\
\hline \hline$-m_{\text {res }}$ & $7.38(12)$ & $3.93(54)$ & $4.19(4)$ \\
\hline \hline
\end{tabular}

Table 2: The RHQ action parameters determined from matching the quantities (1)-(3), and extrapolated to the chiral limit.

\subsection{Heavy-heavy sector: the predictions on bottomonium states}

What would we expect for the size of the errors on the bottomonium masses computed in this way? A naive estimate would be the size of a typical order $a^{2}$ operators, for example, $\hat{O}=$ $\bar{\Psi} \vec{\gamma} \cdot \vec{D} \vec{D}^{2} \Psi$. The typical velocity in bottomonium system $v \sim 0.1$, which is determined by the splitting $\Upsilon(2 S)-\Upsilon(1 S) \sim m v^{2} \sim 500 \mathrm{MeV}$. This indicates $\langle\hat{O}\rangle \sim \frac{p^{4} a^{2}}{m_{b}} \sim 300 \mathrm{MeV}$.

Let's turn to the numerical results given by this work. All calculations are made from a complete calculation of each jackknife block from which the average and errors are determined. The results for various quantities are summarized in Tab. 3.2 and compared to experimental values and other lattice calculations. Our results include only statistical errors. Experimental results are from PDG unless otherwise specified. We can see that most of our calculated bottomonium masses are within $30 \mathrm{MeV}(<1 \%)$ and one standard deviation of the experimental value. Of course, the results for the mass splittings give a more precise test: the spin-orbit splitting from our calculation is about 2.5 standard deviations from the experiment and the hyperfine splitting is further away from the value newly determined by BaBar collaboration [13] or lattice NRQCD calculation [14]. But the accuracy of our results indeed comes as a surprise! We were expecting errors of hundreds $\mathrm{MeV}$ but only see errors several times smaller.

A possible better theoretical estimate would use a simple hydrogen-like Coulomb model to describe bottomonium. In that case, we could define a $\Upsilon$ state as:

$$
\left|\Upsilon, m_{j}\right\rangle=\int \frac{d^{3} \vec{p}_{1} d^{3} \vec{p}_{2}}{(2 \pi)^{9 / 2}} \sum_{s_{1} s_{2}} \delta^{(3)}\left(\vec{P}-\vec{p}_{1}-\vec{p}_{2}\right) \phi\left(\frac{\vec{p}_{1}-\vec{p}_{2}}{2}\right)_{1 S}\left\langle 1 m_{j} \mid s_{1} s_{2}\right\rangle a^{\dagger}\left(\vec{p}_{1}, s_{1}\right) b^{\dagger}\left(\vec{p}_{2}, s_{2}\right)|0\rangle
$$

where $a$ and $b$ are free field quark and anti-quark annihilation operators as in

$$
\Psi(\vec{x})=\int \frac{d^{3} \vec{p}}{\sqrt{2 E_{p}}} \frac{1}{(2 \pi)^{3}} \sum_{s}\left\{u^{s}(p) e^{i \vec{p} \cdot \vec{x}} a(\vec{p}, s)+v^{s}(p) e^{-i \vec{p} \cdot \vec{x}} b^{\dagger}(\vec{p}, s)\right\}
$$




\begin{tabular}{|c|c|c|c|}
\hline \hline quantities & RHQ(MeV) & Exp.(MeV) & NRQCD(MeV) \\
\hline$m_{\eta_{b}}$ & $9420(14)$ & $9389(3)(3)[13]$ & \\
$m_{\Upsilon}$ & $9444(17)$ & 9460 & \\
$m_{\chi_{b 0}}$ & $9873(15)$ & 9859 & \\
$m_{\chi_{b 1}}$ & $9897(16)$ & 9893 & \\
$m_{h_{b}}$ & $9908(17)$ & - & $9900(6)[14]$ \\
$m_{\Upsilon}-m_{\eta_{b}}$ & $23.7(3.7)$ & $71(3)(3)[13]$ & $61(14)[14]$ \\
$m_{\chi_{b 1}}-m_{\chi_{b 0}}$ & $24.0(3.5)$ & 33.34 & \\
\hline \hline
\end{tabular}

Table 3: The predictions on individual masses and splittings, compared to experiment and lattice NRQCD calculations. Results from our calculation include statistical errors only.

and $\phi(\vec{p})$ is the Fourier transform of the hydrogen atom wave function with mass $m / 2$, the reduced mass of the bottomonium system.

The size of the matrix element of the operator $\hat{O}$ can then be estimated and yields the result:

$$
\begin{aligned}
\langle\hat{O}\rangle \sim \frac{5}{8} m_{b}^{3} \alpha_{s}^{4} a^{2}= & \sim 40 \mathrm{MeV} & m_{b}=4.0 \mathrm{GeV}, \alpha_{s}=0.25 \\
& \sim 146 \mathrm{MeV} & m_{b}=4.0 \mathrm{GeV}, \alpha_{s}=0.35
\end{aligned}
$$

Thus the size is very sensitive to the strong coupling constant $\alpha_{s}$. As an accurate $\alpha_{s}$ is not available we can not make any strong statement from this approach.
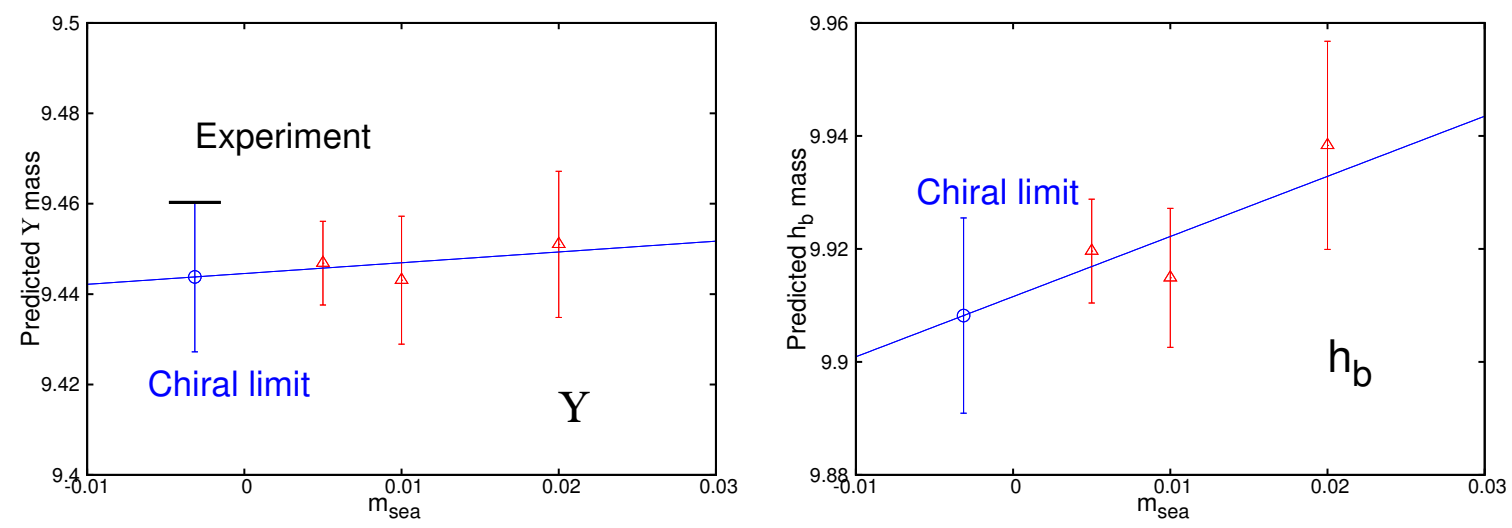

Figure 4: The mass predictions in the chiral limit compared with the experiment. (left) $\Upsilon$; (right) $h_{b}$.

\section{Conclusion}

We have studied the bottomonium and bottom-strange system. We calculate the RHQ parameters by matching the bottom-strange system to the experiment at each sea quark mass and extrapolate to the chiral limit. The bottomonium spectrum is then determined in the chiral limit within $30 \mathrm{MeV}$ of the experimental values. A naive estimate of the size of a $a^{2} \vec{p}^{2}$ operator suggests the error expected in bottomonium spectrum should be a few hundred $\mathrm{MeV}$, while a more careful hydrogen-like Coulomb model suggests the error size is very sensitive to the strong coupling constant $\alpha_{s}$. Other phenomenological models might be useful to estimate the error size more accurately. In summary, our application of the RHQ action on the bottom system yields surprisingly accurate results. Our possible next steps include study of heavy-light system which could 

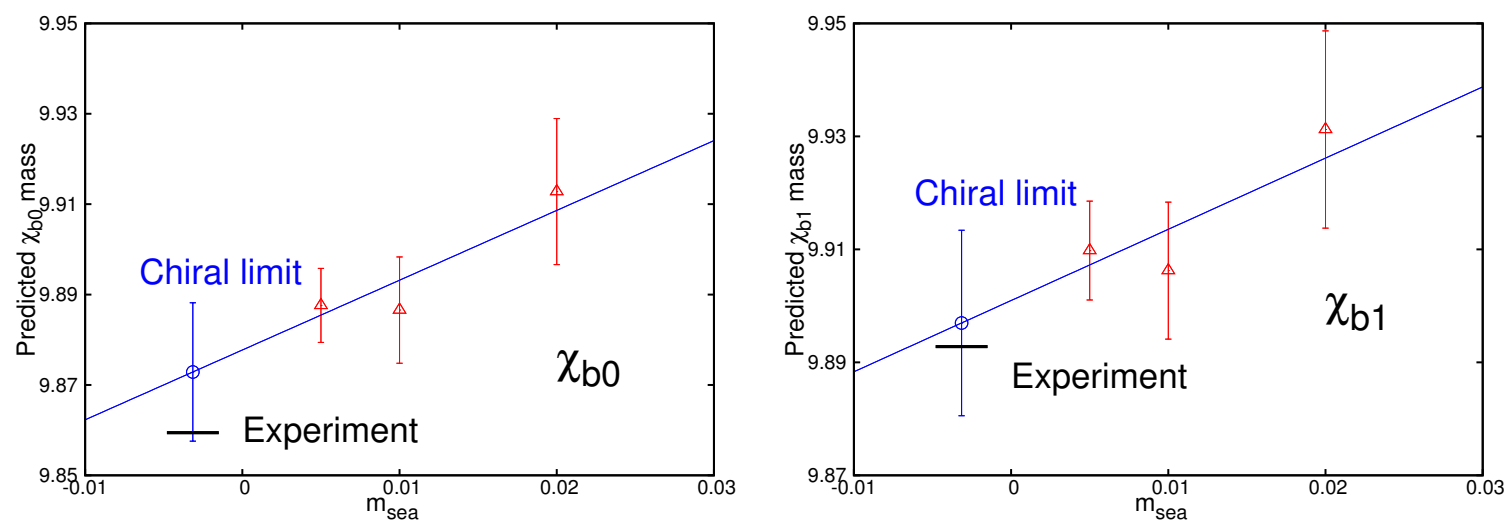

Figure 5: The mass predictions in the chiral limit compared with the experiment. (left) $\chi_{b 0}$; (right) $\chi_{b 1}$.

double-check the validity of the RHQ method in this regime, more calculations on other states like $\bar{b} c$ system and nucleons with one or more heavy quarks, and matrix elements. These calculations could also be repeated using the new $32^{3} \times 64$ lattices to better determine the continuum limit.

\section{Acknowledgment}

We acknowledge helpful discussions with Norman Christ, Robert Mawhinney and Huey-Wen Lin. This work was performed on the QCDOC computers at BNL, Columbia, Edinburgh and the RBRC at BNL, and was supported by U.S. DOE grant DE-FG02-92ER40699.

\section{References}

[1] A. S. Kronfeld, Nucl. Phys. Proc. Suppl. 129, 46 (2004), hep-lat/0310063.

[2] M. Wingate, Nucl. Phys. Proc. Suppl. 140, 68 (2005), hep-lat/0410008.

[3] M. Okamoto, PoS LAT2005, 013 (2006), hep-lat/0510113.

[4] T. Onogi, PoS LAT2006, 017 (2006), hep-lat/0610115.

[5] M. Li and H.-W. Lin, PoS LAT2007, 117 (2007), 0710.0910.

[6] A. X. El-Khadra, A. S. Kronfeld, and P. B. Mackenzie, Phys. Rev. D55, 3933 (1997), hep-lat/9604004.

[7] S. Aoki, Y. Kuramashi, and S.-i. Tominaga, Prog. Theor. Phys. 109, 383 (2003), hep-lat/0107009.

[8] N. H. Christ, M. Li, and H.-W. Lin (2006), hep-lat/0608006.

[9] N. H. Christ, M. Li, and H.-W. Lin, PoS LAT2006, 171 (2006).

[10] H.-W. Lin and N. Christ (2006), hep-lat/0608005.

[11] C. Allton et al. (2008), 0804.0473.

[12] C. Allton et al. (RBC and UKQCD), Phys. Rev. D76, 014504 (2007), hep-lat/0701013.

[13] B. Aubert et al. (BABAR), Phys. Rev. Lett. 101, 071801 (2008), 0807.1086.

[14] A. Gray et al., Phys. Rev. D72, 094507 (2005), hep-lat/0507013. 